\title{
Atividade antiinflamatória do extrato aquoso de Arrabidaea chica (Humb. \& Bonpl.) B. Verl. sobre o edema induzido por venenos de serpentes amazônicas
}

\author{
Denys Paixão Costa de Oliveira, ${ }^{* 1}$ Maria Rosa Lozano Borrás, ${ }^{1}$ Luis Carlos de \\ Lima Ferreira, ${ }^{2}$ Jorge Luis López-Lozano ${ }^{3}$
}

\author{
${ }^{1}$ Faculdade de Ciências Farmacêuticas, Universidade Federal do Amazonas, Rua Comendador \\ Alexandre Amorim, 330, Aparecida, 69010-300 Manaus-AM, Brasil \\ ${ }^{2}$ Departamento de Patologia e Medicina Legal, Faculdade de Medicina, Universidade Federal do \\ Amazonas, Avenida Afonso Pena, 1053, Praça 14, 69000-000 Manaus-AM, Brasil \\ ${ }^{3}$ Centro de Ofidismo "Prof. Paulo Friedrich Bührnheim", Fundação de Medicina Tropical do \\ Amazonas, Avenida Pedro Teixeira, 25, Dom Pedro, 69040-000 Manaus-AM, Brasil
}

\begin{abstract}
RESUMO: O estudo investigou o efeito antiinflamatório do extrato aquoso da Arrabidaea chica (Humb. \& Bonpl.) B. Verl., Bignoniaceae, popularmente conhecida como "crajiru", sobre o edema induzido por venenos de serpentes amazônicas dos gêneros Brothrops e Crotalus, em camundongos albinos, por via oral, intraperitoneal e subcutânea. $\mathrm{O}$ efeito anti-edematogênico foi avaliado pela medição do diâmetro dos coxins das patas posteriores, sendo medidos as 1, 3, 6, 12 e 24 horas, para B. atrox e 1, 3 e 6 horas para Crotalus durissus ruruima, e também avaliado por histopatologia. $\mathrm{O}$ estudo mostrou que o efeito inibitório do extrato aquoso para o gênero Bothrops, pelas vias subcutânea e intraperitoneal (12 horas) foi de $55,87 \%$ e $65,70 \%$, respectivamente. Para o gênero Crotalus o efeito inibitório do extrato pela via subcutânea após 3 horas foi de 33,55\% e após 6 horas de $79,81 \%$. Pela via intraperitoneal após 3 horas foi de $48,02 \%$ e após 6 horas de $92,52 \%$. Na análise histopatológica, o infiltrado de granulócitos e a miocitólise foram os efeitos inflamatórios inibidos mais significativamente. Os resultados sugerem a presença no extrato aquoso de $A$. chica de substâncias com atividade inibitória sobre os efeitos inflamatórios dos venenos das serpentes Bothrops atrox e Crotalus durissus ruruima.
\end{abstract}

Unitermos: Arrabidaea chica, Bignoniaceae, plantas medicinais, venenos, serpentes, atividade antiinflamatória.

\begin{abstract}
Anti-inflammatory activity of the aqueous extract of Arrabidaea chica (Humb. \& Bonpl.) B. Verl. on the self-induced inflammatory process from venoms amazonians snakes". The study investigated the anti-inflammatory effects of the aqueous extract (EAq), the Arrabidaea chica (Humb. \& Bonpl.) B. Verl., Bignoniaceae, known as the "crajiru", tested on the paw oedema induced in inflammatory process by venoms Amazon snakes from the Bothrops and Crotalus species, on albino mice. This process was done by an oral, intraperitoneal and subcutaneous way. The cushions thickness was measured at time breaks of $1,3,6,12$ and 24 hours, Bothrops genus and 1, 3 and 6 hours, Crotalus genus, and a histopathologic analysis was made. The Bothrops genus, was used as an inhibitory effect for the subcutaneous and intraperitoneal way (12 hours), and it had an inhibition of $55.87 \%$ and $65.70 \%$, respectively. For the Crotalus genus, the inhibitory effect for the subcutaneous way after 3 hours was the $33.55 \%$ and $79.81 \%$, after 6 hours. The intraperitoneal way affect after 3 hours had an inhibition of $48.02 \%$ and $92.52 \%$ after 6 hours. For the histopathological analysis, the leucocytes infiltration and the miocitolisis were the most expressive parameters and thus inhibited. The results suggest the presence of active principles with anti-inflammatory effects.
\end{abstract}

Keywords: Arrabidaea chica, Bignoniaceae, medicinal plants, venoms, snakes, anti-inflammatory activity. 


\section{INTRODUÇÃO}

A Flora Amazônica é considerada a maior reserva de biodiversidade do planeta, com poucas espécies de plantas estudadas de forma científica. Diante deste universo de etnoconhecimento botânico, é possível a busca de novas drogas com o estudo de plantas de uso popular consagrado para certa finalidade (Agra et al., 2008; Bertucci et al., 2008; Marliére et al., 2008; Veiga-Junior, 2008; Jesus et al., 2009; Leitão et al., 2009; Santos et al., 2009).

A espécie Arrabidaea chica (Humb. \& Bonpl.) B. Verl., Bignoniaceae, variedade $\mathrm{AC} 2$, é conhecida popularmente como cajuru, carajirú, crajirú, cipó-pau, pariri, cipó-cruz (Cronquist, 1988; Grenand et al., 1987; Pauletti et al., 2003). O uso popular da $A$. chica é feito através do decocto utilizado como antiinflamatório, cicatrizante, em anemias, cólicas intestinais, hemorragia, diarréia, leucorréia e leucemia, a tintura usada para tratar infecções cutâneas, e uso ginecológico realizado através do "banho de assento". Aplicações locais são feitas através de compressas ou banhos (Maia et al., 1994; Costa et al., 1990) e até em cosméticos (Biavatti et al., 2007) Dessa espécie já foram isolados taninos, 7,4-dihidroxi5-metoxiflavona, fitosteróis, flavonóides, pigmentos utilizados em cosméticos como: carajurona e carajurina, compostos derivados das 3-deoxiantocianidinas como a antocianidina, o pigmento 1 , o pigmento 2 e a luteolina e flavona carajuruflavona também foi isolada de Arrabidaea chica (Takemura et al., 1995; Zorn, 2001; Alcerito et al., 2002; Devia et al. 2002). Testes de toxicidade aguda indicaram que a DL50 em camundongos, ultrapassa 2 $\mathrm{g} / \mathrm{kg}$ i.p. e $6 \mathrm{~g} / \mathrm{kg}$ v.o., sugerindo baixa toxicidade do extrato aquoso. Nos testes de toxicidade crônica com as doses avaliadas nenhum sinal ou sintoma anormal, ou alterações histopatológicas foram observados. A atividade imunofarmacológica, in vitro, testada no modelo de pleurisia induzida com zimosan e na ativação in vivo de linfócitos em camundongos, confirmaram atividade antiinflamatória, descrita no uso popular e experimento também sugerem atividade imunoreguladora para os constituintes químicos desta espécie (Oliveira et al., 1995; Chu \& Borrás, 1997) e, Barbosa et al. (2008), reportaram o isolamento de três flavonóides e a inibição total do crescimento de Trichophyton mentagrophytes e um significante efeito tripanocida do extrato etanólico e de suas frações. Não foi detectada qualquer toxicidade aguda relevante, mesmo a uma dose de $1000 \mathrm{mg} / \mathrm{kg}$.

Os venenos de serpentes são conhecidos pelo homem desde os tempos antigos, sendo uma mistura complexa de proteínas e peptídeos. Entre eles estão hemorraginas, proteases, fosfolipases A2 (PLA2) e miotoxinas que atuam por diferentes mecanismos. A composição do veneno pode variar de acordo com a distribuição geográfica e ontogenia nos espécimes de uma mesma espécie (Bolaños, 1984; Assakura et al., 1992; Rodrigues et al., 1998). A classificação dos venenos das serpentes peçonhentas brasileiras, segundo sua atividade fisiopatológica é para o gênero Bothrops e Lachesis: proteolítica, coagulante e hemorrágica, para os gêneros Micrurus: neurotóxica, para o gênero Crotalus: neurotóxica, miotóxica e coagulante (Cardoso, 1990).

Os envenenamentos em humanos causados por serpentes constituem-se num problema de saúde pública, em virtude de sua grande freqüência e gravidade, especialmente onde há falta de recursos terapêuticos, além do pouco conhecimento dos profissionais de saúde relacionado ao tema (Bolaños, 1984; Brasil, 1999). A evolução dos processos inflamatórios nos acidentes ofídicos botrópicos pode levar à necrose tecidual, gangrena, e causar à amputação do membro atingido e/ou a morte do paciente (Barravieira et al., 1994). O tratamento mais eficaz, até agora, para os pacientes de acidentes ofídicos é a soroterapia. Porém, o soro antiofídico neutraliza muito bem o efeito sistêmico do envenenamento, mas não os efeitos locais, que em caso de acidente botrópico e laquético, observa-se um forte processo inflamatório, mas com menor intensidade no caso de acidentes produzidos por espécimes do gênero Crotalus. A reação inflamatória aparece em resposta a estímulo de natureza química, física ou infecciosa. Vários mediadores químicos (Histaminas, 5-HT, Cininas, PGE2, PGF2, PGI2, TXA2, LTB2, PAF, IL1, IL2) são mensageiros biológicos capazes de induzir uma resposta biológica específica (Barreiro \& Lima, 1993). Dados do uso popular e de trabalhos experimentais têm demonstrado a potente atividade antiinflamatória da Curcuma longa e Arrabidaea chica (Melo et al., 2007). Estes dados nos estimularam a testar em camundongos o extrato aquoso desta planta sobre a atividade inflamatória induzida pelas toxinas dos venenos de serpentes de espécies Amazônicas. O objetivo foi avaliar experimentalmente a atividade do extrato aquoso de Arrabidaea chica, sobre o processo inflamatório induzido no coxin plantar de camundongos albinos (Mus musculus) pelos venenos das serpentes amazônicas Bothrops atrox ou Crotalus durissus ruruima.

\section{MATERIAL E MÉTODOS}

A espécie Arrabidaea chica, variedade AC2 foi coletada no setor de plantas medicinais da Empresa Brasileira de Pesquisa Agropecuária, Rodovia AM 010 - KM 28 - município de Manaus-AM, e uma amostra da planta foi depositada no Herbário do Instituto Nacional de Pesquisa da Amazônia - INPA sob o n ${ }^{\circ}$. 214.794. Após identificação botânica, as folhas foram separadas, secas, trituradas e o extrato aquoso (EAq) foi obtido pelo método de Maceração. O EAq foi submetido à liofilização, obtevese $130 \mathrm{~g}$ de material liofilizado.

Foram utilizados camundongos albinos (Mus musculus), com $20 \mathrm{~g}$ de peso, provenientes do Centro de Ofidismo "Prof. Paulo Friedrich Bührnheim" da Fundação de Medicina Tropical do Amazonas - FMT-AM. 
Os animais foram mantidos em gaiolas de polipropileno "mouse pack", sob temperatura controlada entre 22 e 25 ${ }^{\circ} \mathrm{C}$, recebendo água e ração apropriada "ad libitum", e iluminação controlada com ciclo de 12 horas. Os animais foram separados de forma aleatória estratificada. Foram utilizados oito grupos experimentais com doze animais cada, divididos igualmente para os dois venenos testados, ficando quatro grupos, um para cada via de administração e um controle, para cada veneno. Os venenos de serpentes de Crotalus durissus ruruima e Bothrops atrox foram fornecidos pelo Centro de Ofidismo "Prof. Paulo Friedrich Bührnheim" da Fundação de Medicina Tropical do Amazonas - FMT-AM.

\section{Indução do efeito edematogênico dos venenos de serpentes}

Para a determinação da cinética da atividade edematogênica dos venenos, foi empregado o método de Furtado et al. (1991). Grupos de doze camundongos foram injetados subcutaneamente no coxim da pata esquerda (pata experimental) com a dose $(\mu \mathrm{g})$ de veneno diluído em $50 \mu \mathrm{L}$ de solução salina, enquanto, no coxim da pata direita (pata controle) foram injetados apenas $50 \mu \mathrm{L}$ de solução salina. As espessuras dos coxins foram medidas usando-se um micrômetro Mitutoy digital (sensibilidade de $0,01-9,0 \mathrm{~mm}$ ) nos intervalos de tempo de 1, 3, 6, 12 e 24 horas para o teste com o veneno de $B$. atrox e 1, 3 e 6 horas para o teste com veneno de Crotalus d. ruruima. $\mathrm{O}$ edema foi expresso pela diferença entre as espessuras do coxim da pata experimental e controle, dividido pela espessura da pata controle, e multiplicado por 100. Foi construído uma Figura da diferença da espessura da pata experimental com respeito a espessura da pata controle em função do tempo.

\section{Avaliação da atividade antiedematogênica do extrato de A. chica}

Para a avaliação da atividade antiinflamatória do extrato aquoso de $A$. chica foi usado o método de Pereira et al. (1992.) O veneno foi injetado uma hora após a administração do extrato aquoso de $A$. chica. Foram utilizados oito grupos experimentais, divididos igualmente para os dois venenos testados, ficando quatro grupos, um para cada via de administração e um controle, para cada veneno.

As alterações histopatológicas foram analisadas através de cortes histológicos com a espessura de $5 \mu \mathrm{m}$, corados com Hematoxilina \& Eosina. (Prophet et al., 1995). Nas análises dos cortes histológicos foram estabelecidos 5 parâmetros inflamatórios: edema, infiltrado de granulócitos, miocitólise, sufusão hemorrágica e trombo. A intensidade dos parâmetros foi estabelecida em cruz, de acordo com o seguinte critério: Leve (+); Moderado (++); Acentuado $(+++)$. Para a análise estatística foi utilizada a análise de variância ANOVA e o teste " $\mathrm{t}$ ", com níveis de significância de $5 \%(p<0,05)$.

\section{RESULTADOS E DISCUSSÃO}

Segundo a Figura 1, o extrato aquoso de $A$. chica sobre a atividade inflamatória do veneno de $B$. atrox, pelas vias subcutânea e intraperitoneal (12 horas), mostrou atividade inibitória de $55,87 \%$ e $65,70 \%$, respectivamente. Com relação a atividade inflamatória do veneno de Crotalus, o extrato aquoso de $A$. chica pela via subcutânea, às 3 horas foi de $33,55 \%$ e às 6 horas de $79,81 \%$, e pela via intraperitoneal esta atividade inibitória às 3 horas foi de $48,02 \%$ e às 6 horas de $92,52 \%$ respectivamente (Figura 2). Mas o extrato aquoso de $A$. chica, com as doses avaliadas por via oral, não apresentou efeito inibitório sobre a atividade inflamatória dos venenos de $B$. atrox e $C$. d. ruruima.

$\mathbf{A}$

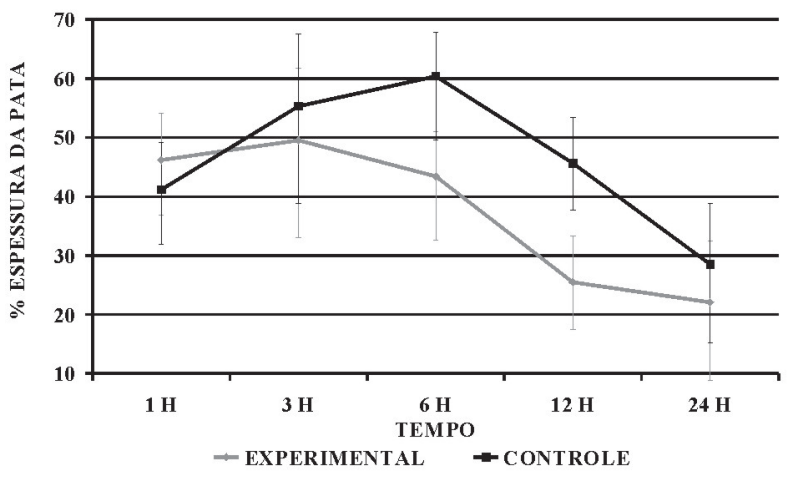

B

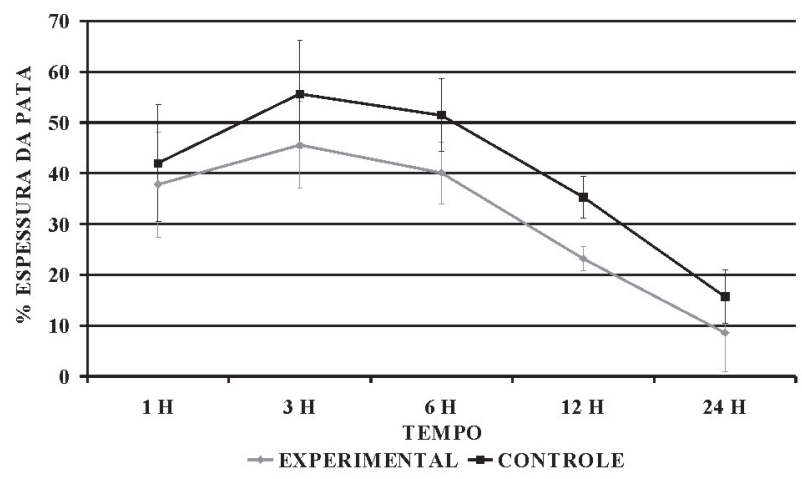

Figura 1. Efeito da atividade antiinflamatória do extrato aquoso A. chica induzido por $5 \mu \mathrm{g}$ do veneno de Bothrops atrox $(\mathrm{n}=$ 12/grupo). (A) via subcutânea $(212,5 \mathrm{mg} / 500 \mu \mathrm{L})$ e (B) via intraperitoneal $(50 \mathrm{mg} / 500 \mu \mathrm{L})$. 
A

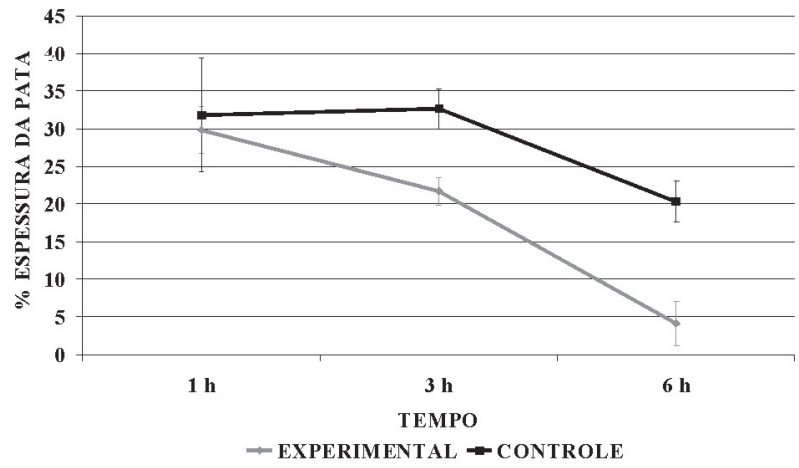

B

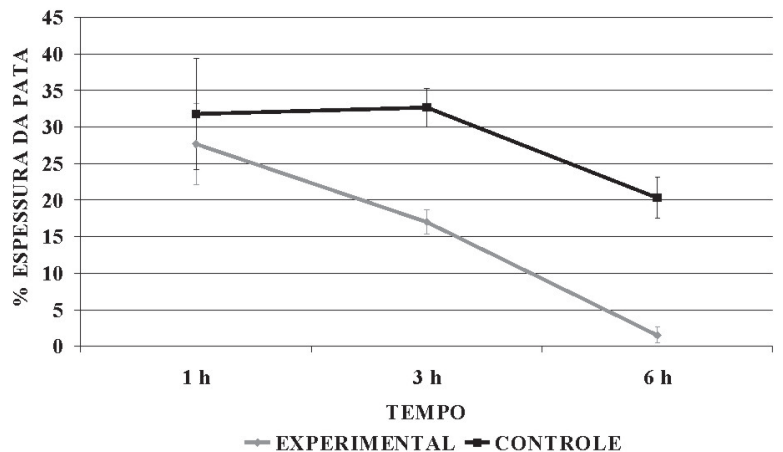

Figura 2. Efeito da atividade antiinflamatória do extrato aquoso $A$. chica induzido por $\mu$ g Crotalus durissus ruruima (n = 8/grupo). (A) via subcutânea $(212,5 \mathrm{mg} / 500 \mu \mathrm{L})$ e (B) via intraperitoneal $(50 \mathrm{mg} / 500 \mu \mathrm{L})$.

$\mathrm{Na}$ análise histopatológica dos parâmetros analisados, o infiltrado de granulócitos e a miocitólise foram os mais significativamente inibidos pelo extrato aquoso de $A$. chica (Figuras 3, 4, 5 e 6).

A

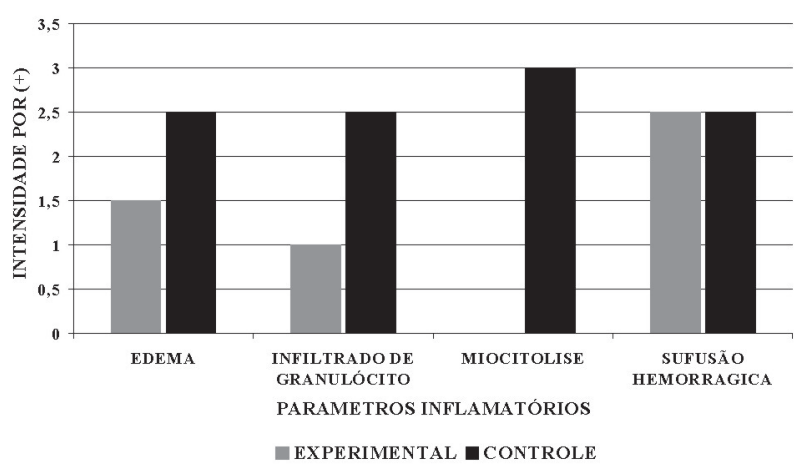

Figura 3. Análise histopatológica do efeito do extrato aquoso A. chica, por via subcutânea, sobre o processo inflamatório após (A) 12 horas de inoculação de $5 \mu \mathrm{g}$ do veneno de Bothrops atrox.

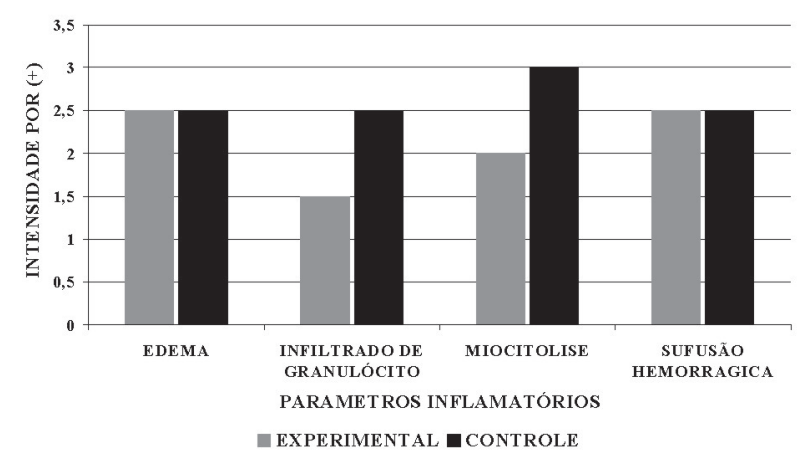

Figura 4. Análise histopatológica do efeito do extrato aquoso A. chica, por via intraperitonial, sobre o processo inflamatório após (A) 12 horas de inoculação de $5 \mu \mathrm{g}$ do veneno de Bothrops atrox.

$\mathbf{A}$

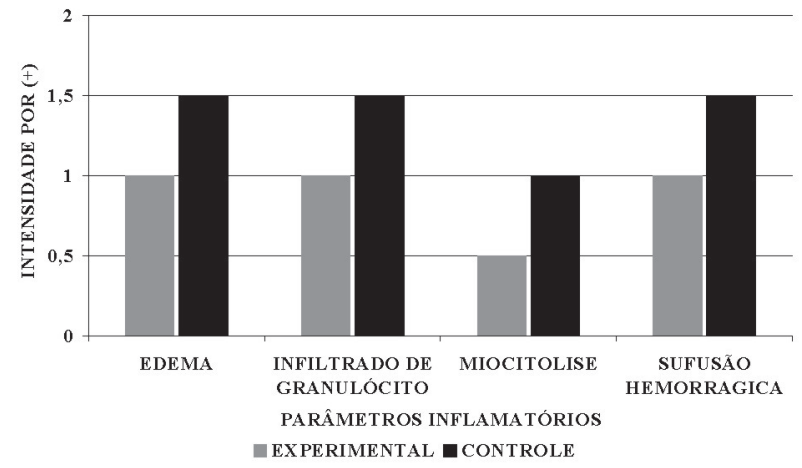

B

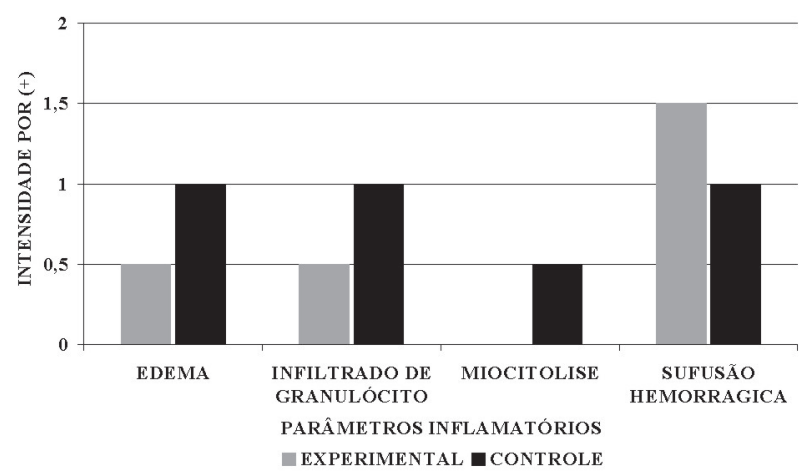

Figura 5. Análise histopatológica do efeito do extrato aquoso $A$. chica, por via subcutânea, sobre o processo inflamatório após (A) 1 hora; (B) 6 horas de inoculação de $5 \mu \mathrm{g}$ do veneno de Crotalus durissus ruruima. 
A

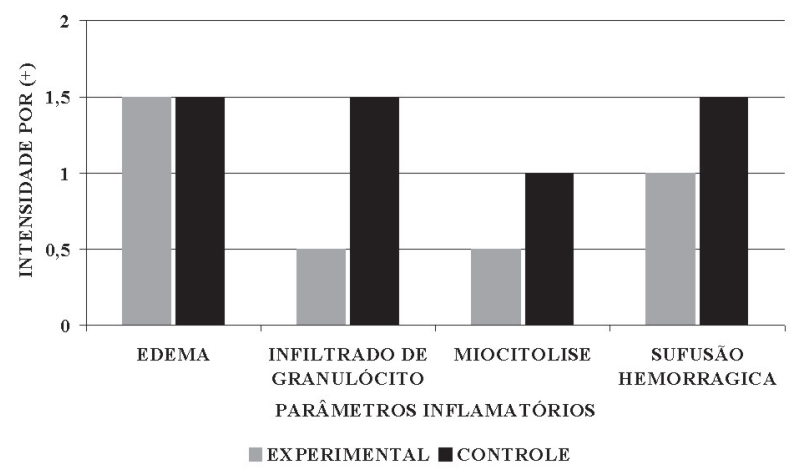

B

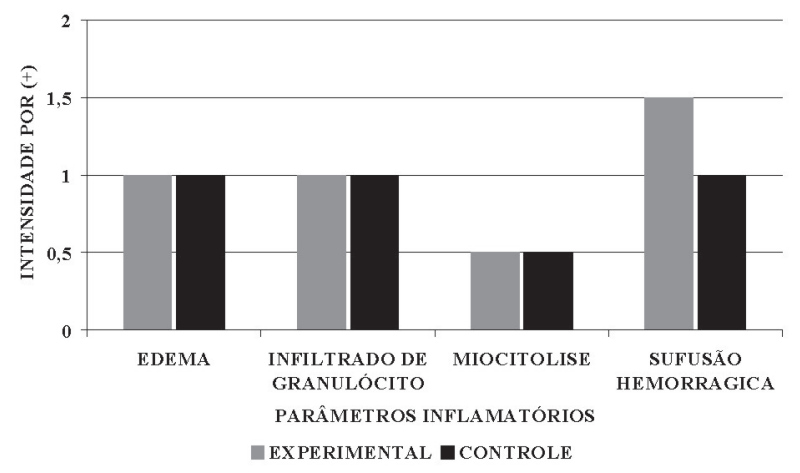

Figura 6. Análise histopatológica do efeito do extrato aquoso A. chica, por via intraperitoneal, sobre o processo inflamatório após (A) 1 hora; (B) 6 horas de inoculação de $5 \mu \mathrm{g}$ do veneno de Crotalus durissus ruruima.

\section{DISCUSSÃO}

Os venenos de serpentes da família Viparidae possuem potente ação inflamatória, desencadeada rapidamente após inoculação do veneno, afetando drasticamente o tecido muscular, vasos sangüíneos e pele, induzindo lesões que podem levar à seqüelas e geralmente causam infecções, dificultando o manejo do quadro (Gutierrez \& Lomonte, 2003).

Neste trabalho utilizaram-se como agentes indutores da inflamação, nos coxins plantares de camundongo, os venenos de B. atrox ou C. d. ruruima, sendo observada a presença de processos inflamatórios associados com hemorragia. A atividade máxima de indução do processo inflamatório foi observada às 3 horas após a inoculação de ambos os venenos, embora o processo inflamatório tivesse uma duração de 24 horas quando induzido pelo veneno de $B$. atrox e só de 6 horas quando induzido pelo veneno de $C . d$. ruruima. As alterações histopatológicas produzidas pelas ações dos venenos botrópicos são conseqüências de sua ação pró-inflamatória, proteolítica, hemorrágica e coagulante. As lesões no local de inoculação do veneno variam grandemente de intensidade (Benvenuti, 2003).

Quando o extrato aquoso de $A$. chica foi adminsitrado pela via oral, não foi observada atividade inibitória sobre a inflamação induzida por ambos os venenos. $\mathrm{Na}$ análise histopatológica dos coxins das patas dos camundongos dos grupos experimentais, quando extrato aquoso de $A$. chica foi administrado por via intraperitoneal ou subcutânea, observouse que houve uma atividade inibitória principalmente sobre os processos de miocitólise e de migração de granulócitos induzidos pelos venenos de $B$. atrox ou $C$. d. ruruima, o que sugere uma possível ação de substância(s) presente no extrato aquoso de $A$. chica inibindo toxinas que induzem estes dois processos próinflamatórios e/ou inibindo fatores endógenos que estimulam ambos os processos pró-inflamatórios. Esta(s) substância(s) não seria (m) absorvida(s) por via oral, visto não terem sido observados efeitos por esta via e/ou podem sofrer transformações químicas por processos enzimáticos, no trato intestinal, durante o processo de absorção e/ou no fígado, alterando a sua função inibitória. Os processos pró-inflamatórios de edema e sufusão hemorrágica induzidos pelos venenos de $B$. atrox ou $C$. d. ruruima não foram inibidos pelas doses do extrato aquoso de $A$. chica testadas, independente da via administrada.

O óxido nítrico (NO) reconhecido no momento como mediador que causa vasodilatação através do fator relaxante derivado do endotélio (EDRF), é produzido pela célula endotelial. No processo inflamatório, além de produzir vasodilatação e formação de edema, também promove a biossíntese de prostanóides decorrente da ativação da COX-2 (Landucci, 1996). Estudo anterior mostrou que o extrato aquoso do A. chica foi eficaz em inibir a produção de óxido nítrico por macrófagos elicitados por tioglicolato, estimulados por lipopolisacarídeo bacteriano (LPS) in vitro. Portanto, a inibição da migração de granulócitos diminuindo assim a inflamação de mediadores próinflamatórios, sugere um dos mecanismos moleculares pelos quais os princípios ativos da $\mathrm{A}$. chica apresentaram atividade inibitória do processo inflamatório induzido pelos venenos das serpentes utilizadas.

Dentre os metabólitos de planta, os flavonóides são provavelmente os mais versáteis. Os flavonóides têm sido considerados como responsáveis pela atividade antiinflamatória, anti-hepatotóxica, anti-hipertensiva, e muitas outras atividades, como a inibição de atividade enzimática, com ação inibitória da atividade das fosfolipases A2, um importante componente dos venenos de serpentes (Alcaraz \& Holut, 1985; Mors et al., 2000). A presença de flavonóides em $A$. chica pode ser um dos fatores moleculares importante com atividade anti-fosfolipásica A2 dos venenos de B. atrox e Crotalus d. ruruima, desta forma contribuindo ao efeito inibitório do extrato aquoso sobre a atividade inflamatória induzida pelos venenos de $B$. atrox e Crotalus $d$. ruruima. Os dados experimentais sugerem que o extrato aquoso de A. chica apresenta substâncias químicas com atividade inibitória do processo inflamatório induzido pelos venenos de Bothrops atrox ou Crotalus durissus ruruima quando administrados pelas vias subcutânea $(10,6 \mathrm{~g} / \mathrm{kg}$ peso) e via intraperitoneal $(2,5 \mathrm{~g} / \mathrm{kg}$ de peso), não apresentando efeito por via oral. Dos parâmetros do processo inflamatório avaliados, o extrato aquoso de Arrabidaea chica mostrou atividade inibitória para o infiltrado de granulócitos e miocitólise induzidos pelas toxinas dos venenos de serpentes. Novos experimentos deverão ser realizados para esclarecer o mecanismo de ação desta planta. O isolamento e elucidação dos responsáveis pelo efeito inibitório estão em curso. 
Os resultados obtidos sugerem potencial biotecnológico do extrato aquoso de Arrabidaea chica para obtenção de princípios ativos antiinflamatórios.

\section{AGRADECIMENTOS}

Ao Dr. José Jackson Bacelar Nunes Xavier e Dr. Francisco Célio Maia Chaves, EMBRAPA-AM, pela colaboração e orientação na coleta do material, Ao Dr. Adrian Martin Pohlit, INPA, pela colaboração e orientação na obtenção do extrato, Ao Prof. Nelson Fraiji e Prof ${ }^{a}$ Júlia Ignez Salem, coordenadores do Curso de Mestrado Multidisciplinar em Patologia Tropical.

\section{REFERÊNCIAS}

Agra MF, Silva KN, Basílio IJLD, França PF, Barbosa-Filho JM 2008. Survey of medicinal plants used in the region Northeast of Brazil. Rev Bras Farmacogn 18: 472-508.

Alcaraz MJ, Hoult JRS 1985. Effects of hypolaetin-8-glucoside and related flavonoids on soybean lipoxygenase and snake venom phospholipase $\mathrm{A}_{2}$ Arch Int Pharmacodyn 278: 4-12.

Alcerito T, Barbo FE, Negri G. Santos DYAC, Meda CI, Young MCM, Chávez D, Blatt CTT 2002. Folicular epicuticular wax of Arrabidaea brachypoda: flavonoids and antifungical activity. Biochem Syst Ecol 30: 677-83.

Assakura MT, Furtado MF, Mandelbaum FR 1992. Biochemical and Biological differentiation of the venoms of the lancehead vipers (Bothrops atrox, Bothrops asper, Bothrops marajoensis and Bothrops moojeni). Comp Biochem PhysB 102: 727-32.

Barravieira B. 1994. Venenos animais: uma visão integrada. Rio de Janeiro: Biomédicas.

Barbosa WLR, Pinto LN, Quignard E, Vieira JMS, Silva Jr. JOC, Albuquerque S 2008. Arrabidaea chica (HBK) Verlot: phytochemical approach, antifungal and trypanocidal activities. Rev Bras Farmacogn 18: 544-548.

Barreiro EJ, Lima MEF 1993. Antiinflamatórios Não-Esteróides Parte I. Infarma 2: 6-9.

Benvenuti LA 2003. Anatomia Patológica nos Accidentes por Animais Peçonhentos. In: Cardoso JLC, França FOS, Wen FH, Málaque CMS, Junior VH (Org.). Animais Peçonhentos no Brasil: Biologia, clínica e terapêutica dos acidentes. São Paulo: Savier, p. 347-55..

Bertucci A, Haretche F, Olivaro C, Vázquez A, 2008. Prospección química del bosque de galería del río Uruguay. Rev Bras Farmacogn 18: 21-25.

Biavatti M, Marensi V, Leite SN, Reis A 2007. Ethnopharmacognostic survey on botanical compendia for potential cosmeceutic species from Atlantic Forest. Rev Bras Farmacogn 17: 640-653.

Bolaños R 1984. Serpientes, Venenos y Ofidismo en Centro América. San José: Universitária de Costa Rica.

Brasil 1999. Ministério da Saúde. Manual de diagnóstico e tratamento de acidentes por animais peçonhentos.

Cardoso JLC 1990. Ofidismo, Aracneísmo, Escorpionismo.
Epidiomiologia. Patogenia e Clínica. Diagnostico e Terapêutica. In: SORENSEN, B. Animais Peçonhentos. Rio de Janeiro: Atheneu.

Chu VM, Borrás MRL 1997. Estudo de plantas da Amazônia Ocidental com atividade cicatrizante. Relatório de Pesquisa - Curso de farmácia - Universidade Federal do Amazonas.

Costa PRC 1990. Plantas medicinais nativas e aclimatadas da região Amazônica. Manaus: FUA/LBA.

Cronquist A 1998. An Integral System of Classification of Flowering Plants: The Evolution and Classification of Flowering Plants. New York: Columbia University Press.

Devia B, Llabres G, Wouters J, Wouters J, Dupont L, Escribano-Bailon MT, Angenot L, Tist M 2002. New 3-Deoxyanthocyanidins from Leaves of Arrabidaea chica. Phytochem Analysis 13: 114-120.

Grenand PA, Moretti CA, Jaquemin H 1987. Pharmacopées tradicionnelles en Guyane; créoles, Palikus, Wayãpi. Paris: ORSTOM.

Gutiérrez JM, Lomonte B 2003. Efectos locales en el envenenamiento ofídico en América latina. In: Cardoso JLC, França FOS, Wen FH. Animais Peçonhentos no Brasil: biologia, clínica e terapêutica dos acidentes. São Paulo: Savier.

Furtado MFD, Dias da Silva W, Colleto GMDD 1991. Controle de qualidade dos venenos animais e dos correspondentes antivenenos. I Padronização dos métodos de ensaio das atividades bioquímicas e farmacológicas dos venenos de algumas espécies do gênero Bothrops e Crotalus usando amostras secas a temperaturas ambiente ou liofilizadas. Mem Inst Butanta 53: 149-59.

Jesus NZT, Lima JCS, Silva RM, Espinosa MM, Martins DTO 2009.Levantamentoetnobotânicodeplantaspopularmente utilizadas como antiúlceras e antiinflamatórias pela comunidade de Pirizal, Nossa Senhora do LivramentoMT, Brasil. Rev Bras Farmacogn 19: 130-139.

Landucci ECT 1996. Envolvimento das fosfolipases do tipo $\mathrm{A}_{2}$ (PLA2) no processo inflamatório. Atividade antiinflamatória da Crotapotina. Rio de Janeiro. Fundação Osvaldo Cruz, Tese de Doutorado - Fundação Osvaldo Cruz.

Leitão F, Fonseca-Kruel VS, Silva IM, Reinert F 2009. Urban ethnobotany in Petrópolis and Nova Friburgo (Rio de Janeiro, Brazil). Rev Bras Farmacogn 19: 333-342.

Maia VC; Brandao CIF; Antony RC 1994. Estudo de plantas da Amazônia Ocidental com aplicação em Dermatologia. Reunião Anual da Sociedade Brasileira para o Progresso da Ciência, Vitória. Brasil.

Marliére LDP, Ribeiro AQ, Brandão MGL, Klein CH, Acurcio FA 2008. Utilização de fitoterápicos por idosos: resultados de um inquérito domiciliar em Belo Horizonte (MG), Brasil. Rev Bras Farmacogn 18 (Supl.): 754-760.

Melo MM, Lúcia M, Gerhard G, Habermehl GG 2007. Plant extracts for topic therapy of Bothrops alternatus envenomation. Rev Bras Farmacogn 17: 29-34. 
Mors WB, Nascimento C, Pereira BMR, Pereira NA 2000. Plant natural products active against snake bite - the molecular approach. Phytochemistry 55: 627-642.

Oliveira DPC, Matsuura MM, Borrás MRL 1995. Estudo da atividade biológica da Arrabidaea chica Verl. Crajiru. Relatório de Pesquisa, Curso de Farmácia, Universidade Federal do Amazonas.

Pauletti PM, Bolzani VS, Young MCM 2003. Constituintes químicos de Arrabidaea samydoides (Bignoniaceae). Quim Nova 26: 641-643.

Pereira BMR, Gonçalves LC, Pereira NA 1992. Abordagem farmacológica de plantas recomendadas pela medicina folclórica como antiofídicos III - Atividade antiedematogênica. Rev Bras Farm 73: 85-86.

Prophet EB, Mills B, Arrington JB, Sobin LH 1995. Métodos Histotecnológicos. Instituto de Patologia de las Fuerzas Armadas de los Estados Unidos de América (AFIP): Registro de Patologia de los Estados Unidos de América (ARP), Washington, D.C.

Rodrigues VM, Soares AM, Mancin AC, Fontes MRM, HomsiBrandeburgo MI, Giglio JR 1998. Geographic variations in the composition of myotoxins from Bothrops neuwiedi snake venoms: Biochemical characterization and biological activity. Comp Biochem Physiol A 121: 215222.

Santos EB, Dantas GS, Santos HB, Diniz MFFM, Sampaio FC 2009. Estudo etnobotânico de plantas medicinais para problemas bucais no município de João Pessoa, Brasil. Rev Bras Farmacogn 19: 321-324.

Takemura OS, Linuma M, Tosa H, Miguel OG, Moreira EA, Nozawa Y 1995. A Flavone from leaves of Arrabidaea chica f. CUPREA. Phytochemistry 38: 1299-1300.

Veiga-Junior VF 2008. Estudo do consumo de plantas medicinais na Região Centro-Norte do Estado do Rio de Janeiro: aceitação pelos profissionais de saúde e modo de uso pela população. Rev Bras Farmacogn 18: 308-313.

Zorn B, Garcia-Piñeres AJ, Castro V, Murillo R, Mora G, Merfort I 2001. 3- Desoxyanthocyanidins from Arrabidaea chica. Phytochemistry 56: 831-835. 